\title{
Resonance-enhanced optical annealing of silicon nanowires
}

\author{
Guo-Hui Ding, ${ }^{1,2}$ C. T. Chan, ${ }^{1, *}$ Z. Q. Zhang, ${ }^{1}$ and P. Sheng ${ }^{1}$ \\ ${ }^{1}$ Department of Physics, The Hong Kong University of Science and Technology, Clear Water Bay, Kowloon, Hong Kong, China \\ ${ }^{2}$ Department of Physics, Shanghai Jiao Tong University, Shanghai 200030, China
}

(Received 7 February 2005; published 2 May 2005)

\begin{abstract}
We consider the visible-light absorption by Si nanowires. Because of the cylindrical geometry of the nanowire, there is an $S$-wave resonance mode with $m=0$, which greatly enhances the visible-light absorption of $\mathrm{Si}$ nanowire with diameters of about $20 \mathrm{~nm}$. The energy dissipations by thermal radiation and heat convection are also discussed. Our results give a quantitative explanation using electromagnetic theory of recent experimental phenomena that Si nanowires can ignite in air after a photographic flash.
\end{abstract}

DOI: 10.1103/PhysRevB.71.205302

PACS number(s): 78.20.Ci, 42.25.Bs, 78.20.Nv

\section{INTRODUCTION}

Si nanowires have attracted much research attention in recent years because of their quasi-one-dimensional properties and their potential applications in electronic nanodevices. ${ }^{1}$ Nanomaterials have shown many interesting and sometimes unexpected properties due to their unique structure and low dimensions. One recent example reported by experiment is that $\mathrm{Si}$ nanowires ignite in air when exposed to a conventional photographic flash, and evaporate when flashed in vacuum. ${ }^{2}$ It indicates that a rather high temperature $\left(T>1500{ }^{\circ} \mathrm{C}\right)$ within the $\mathrm{Si}$ cores is achieved, and that $\mathrm{Si}$ nanowires have a strong ability to absorb visible light. It is also found that $\mathrm{Si}$ nanowires with diameters larger than $40 \mathrm{~nm}$ cannot be easily ignited by the conventional photographic flash. This phenomenon is unusual, since the optical absorption coefficients of $\mathrm{Si}$ are low for the wavelength of the incident light ranging from $400 \mathrm{~nm}$ to $7 \mu \mathrm{m}$. A previous estimate $^{2}$ indicates that the temperature increase by photoenergy absorbed from the incident light is not high enough to ignite the Si nanowires. It was thus argued that the mechanism of ignition may be related to quantum confinement effects in the nanowire system. A similar photoeffect has also been observed in single-wall carbon nanotubes (SWNTs). ${ }^{3}$ SWNTs burned away in air after flashing and form mainly $\mathrm{CO}_{2}$ gas. For SWNTs, it was hypothesized that the ignition arises from photophysical effects associated with a metal catalyst and carbon in chemical contact. ${ }^{4,5}$ In this paper, we shall see that Si nanowire ignition is a classical phenomenon and can be explained by considering the Maxwell equations.

\section{THE ABSORPTION AND SCATTERING PROPERTIES OF SI NANOWIRES}

The Si nanowires will be represented by infinite length cylinders since their lengths are very long compared with their diameters. Consider a monochromatic beam of incident light scattered by a cylinder of radius $a$. In the normal incidence and $S$-wave case (TM mode), the electric field $\mathbf{E}$ is parallel to the axis of the cylinder. The total field outside the cylinder is the sum of the incident and scattered fields, given by $\mathbf{E}=\Sigma_{m} i^{m} \exp (\operatorname{im} \theta)\left[J_{m}\left(k_{0} r\right)+D_{m} H_{m}^{(1)}\left(k_{0} r\right)\right] \hat{\mathbf{e}}_{z}$, where $J_{m}$ and $H_{m}^{(1)}$ are the Bessel function and Hankel function of the first kind, respectively, and $\hat{\mathbf{e}}_{z}$ is the unit vector in the axis direction. The total scattering cross section per unit length of the cylinder is given by ${ }^{6}$

$$
\sigma_{\text {scatt }}=\frac{4}{k_{0}} \sum_{m=-M}^{M}\left|D_{m}\right|^{2},
$$

and the total absorption cross section per unit length is

$$
\sigma_{a b s o r b}=-\frac{4}{k_{0}} \sum_{m=-M}^{M}\left[\operatorname{Re}\left(D_{m}\right)+\left|D_{m}\right|^{2}\right],
$$

where $k_{0}$ is the wave vector for the light in air. The scattering coefficients $D_{m}$ can be written as

$$
D_{m}=\frac{k J_{m}\left(k_{0} a\right) J_{m}^{\prime}(k a)-k_{0} J_{m}^{\prime}\left(k_{0} a\right) J_{m}(k a)}{k_{0} H_{m}^{(1)^{\prime}}\left(k_{0} a\right) J_{m}(k a)-k H_{m}^{(1)}\left(k_{0} a\right) J_{m}^{\prime}(k a)}
$$

with $k=k_{0} \sqrt{\epsilon}$ being the wave vector of light in the cylinder.

For the $P$-wave (TE mode) case at normal incidence, the magnetic field $\mathbf{H}$ is parallel to the axis of the cylinder and is given by $\mathbf{H}=\Sigma_{m} i^{m} \exp (\operatorname{im} \theta)\left[J_{m}\left(k_{0} r\right)+D_{m} H_{m}^{(1)}\left(k_{0} r\right)\right] \hat{\mathbf{e}}_{z}$ in the region outside the cylinder. The scattering coefficients $D_{m}$ can be expressed as

$$
D_{m}=\frac{k_{0} J_{m}\left(k_{0} a\right) J_{m}^{\prime}(k a)-k J_{m}^{\prime}\left(k_{0} a\right) J_{m}(k a)}{k H_{m}^{(1)^{\prime}}\left(k_{0} a\right) J_{m}(k a)-k_{0} H_{m}^{(1)}\left(k_{0} a\right) J_{m}^{\prime}(k a)} .
$$

In the following calculations, we take measured values of the complex and frequency dependent permittivity of bulk $\mathrm{Si}$ crystal $^{7}$ in the visible-light wavelength range. Since the Si nanowires that burn under illumination are of 20-nm diam, the dielectric function can be taken as that of bulk Si.

The ability of nanowires to absorb or scatter incident light can be characterized by the absorption and scattering efficiencies, $Q_{\text {scatt }}=\sigma_{\text {scatt }} / G$ and $Q_{\text {absorb }}=\sigma_{\text {absorb }} / G$, where $G$ is the geometric cross section of the cylinder per unit length $(G=d \equiv 2 a)$. We expect that both the dispersive permittivity and the structural resonance will contribute to the wavelength dependence of $Q_{a b s o r b}$ and $Q_{\text {scatt }}$. In Fig. 1 we plot $Q_{a b s o r b}$ as a function of the wavelength for several different diameters of the wires. For the $S$-wave case, there is a broad peak of absorption efficiency around wavelength $\lambda_{0}$ $=350 \mathrm{~nm}$ for the wire with diameter $d=20 \mathrm{~nm}$, and for a 


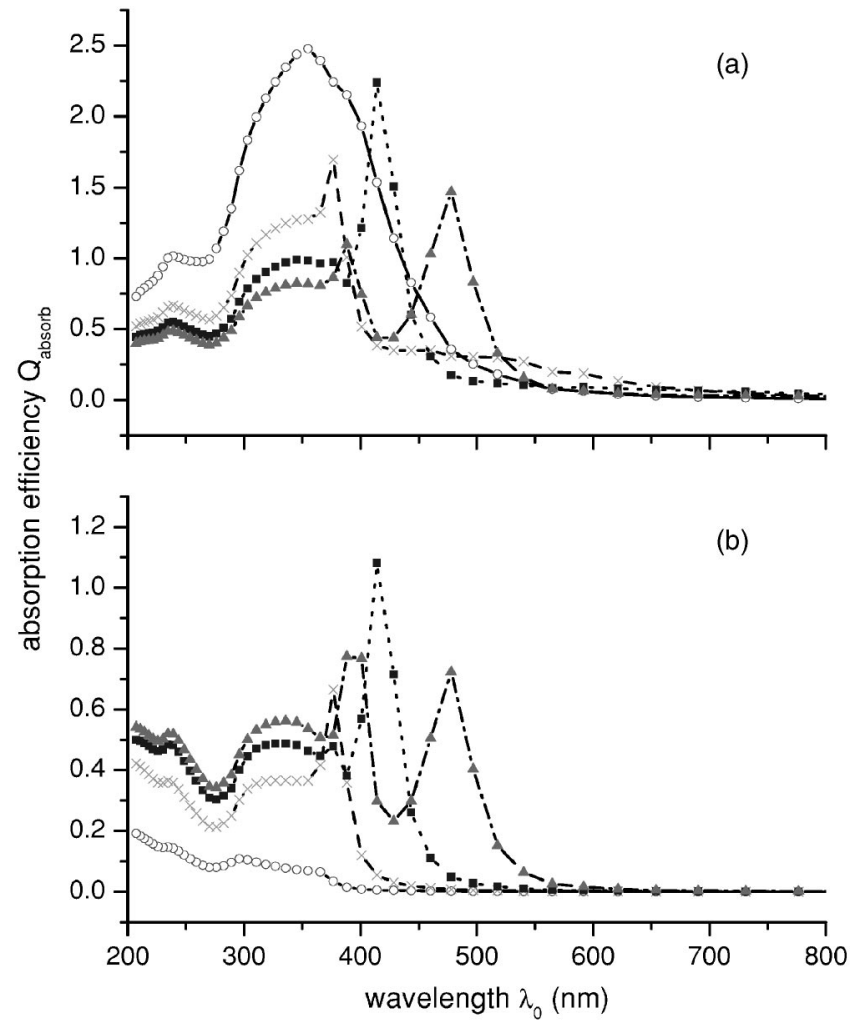

FIG. 1. The absorption efficiency $Q_{a b s o r b}$ of Si nanowire vs the incident light wavelength $\lambda_{0}$ for the cases (a) $S$ wave and (b) $P$ wave. The diameter of nanowire $d=20 \mathrm{~nm}$ (open circles), $40 \mathrm{~nm}$ (crosses), $60 \mathrm{~nm}$ (squares), and $80 \mathrm{~nm}$ (triangles).

wavelength larger than $500 \mathrm{~nm}, Q_{a b s o r b}$ is small. As the diameter of the wire increases, the absorption peak position shifts to the long-wavelength regime. The maximum absorption cross section is about 2.5 times that of the geometric cross section of the wire. In the case of the $P$ wave, the absorption efficiency for the wire with diameter $d=20 \mathrm{~nm}$ is much smaller than the $S$-wave case. When the diameter of the wire increases, $Q_{a b s o r b}$ for the $P$ wave is enhanced and its peak position also shifts to the long-wavelength regime. So for large diameter Si wires, both $S$ and $P$ waves of incident light can be absorbed by the wire significantly, but light absorption is dominated by the $S$ wave for small diameter wires. In Fig. 2, the scattering efficiency $Q_{\text {scatt }}$ as a function of the wavelength is plotted. We see that $Q_{\text {scatt }}$ has a similar peak structure as the $Q_{\text {absorb }}$ in the $P$-wave case. In the $S$-wave case, in addition to the resonance peak structures there are also broad oscillations which arise from the interference effect. ${ }^{6}$

The mechanism of light absorption and scattering can be understood more clearly by fixing the wavelength of incident light and consequently the corresponding permittivity of the $\mathrm{Si}$ wire at this wavelength. In Figs. 3(a) and 3(b) the absorption and scattering efficiency $Q_{\text {absorb }}$ and $Q_{\text {scatt }}$ for $h v$ $=2.5 \mathrm{eV}\left(\lambda_{0} \approx 500 \mathrm{~nm}\right)$ as a function of the diameter of wire is shown. We see that the absorption efficiency oscillates with the variation of the diameter $d$. The first absorption peak for the $S$ wave is near $d=30 \mathrm{~nm}$, and the first absorption peak for the $P$ wave is around $d=85 \mathrm{~nm}$. The first peak of

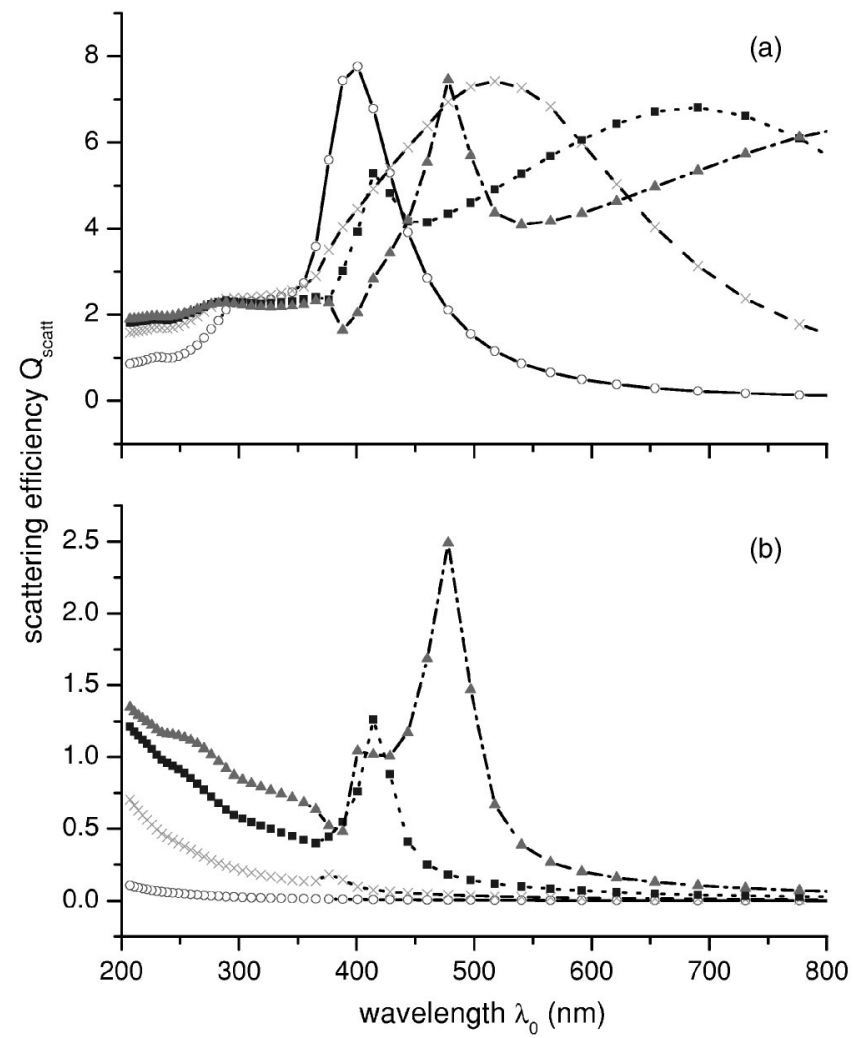

FIG. 2. The scattering efficiency $Q_{\text {scatt }}$ of Si nanowire vs the incident light wavelength $\lambda_{0}$ for the cases (a) $S$ wave and (b) $P$ wave. The diameter of nanowire $d=20 \mathrm{~nm}$ (open circles), $40 \mathrm{~nm}$ (crosses), $60 \mathrm{~nm}$ (squares), and $80 \mathrm{~nm}$ (triangles).

absorption for the $S$ wave is due to the $m=0$ partial-wave mode. There is a Mie resonance for the $m=0$ mode at the diameter $d=30 \mathrm{~nm}$, which is absent for the $P$ wave. The first absorption peak in the $P$-wave case is due to a $m=1$ resonance, and it coincides with the position of the second peak in the $S$-wave case. The existence of resonance mode in partial wave can be revealed further by studying the scattering cross section. The scattering efficiency $Q_{\text {scatt }}$ shows a similar structure of oscillations with the diameter $d$, with essentially the same peak positions as $Q_{a b s o r b}$. We find that the peak structure of $Q_{\text {scatt }}$ does not change significantly even if we take the imaginary part of permittivity to be zero. Therefore, it shows that the absorption of light at $d=20 \mathrm{~nm}$ is indeed enhanced by resonance. In Fig. 3(c) we plot the resonant diameters of the Si nanowire in the visible-light regime for the $m=0$ and $m=1$ modes, respectively. It shows that for small diameter nanowires $(d<50 \mathrm{~nm})$, the $m=0$ mode is the only mode that contributes to resonance in the visible-light wavelength regime. It explains why small diameter nanowires absorb more incident light of $S$-wave polarization than $P$-wave polarization as shown in Fig. 1. The absorption peaks for both $S$ - and $P$-wave cases for diameters $d=60$ and $80 \mathrm{~nm}$ in Fig. 1 can be interpreted as the result of $m=1$ mode resonance. We also have investigated the scattering and absorption of light by a spherical particle of $\mathrm{Si}$. According to the Mie theory, the most important resonances in this case are the electric or magnetic dipole modes with partial-wave number $l=1$. Our calculation reveals that for the incident 


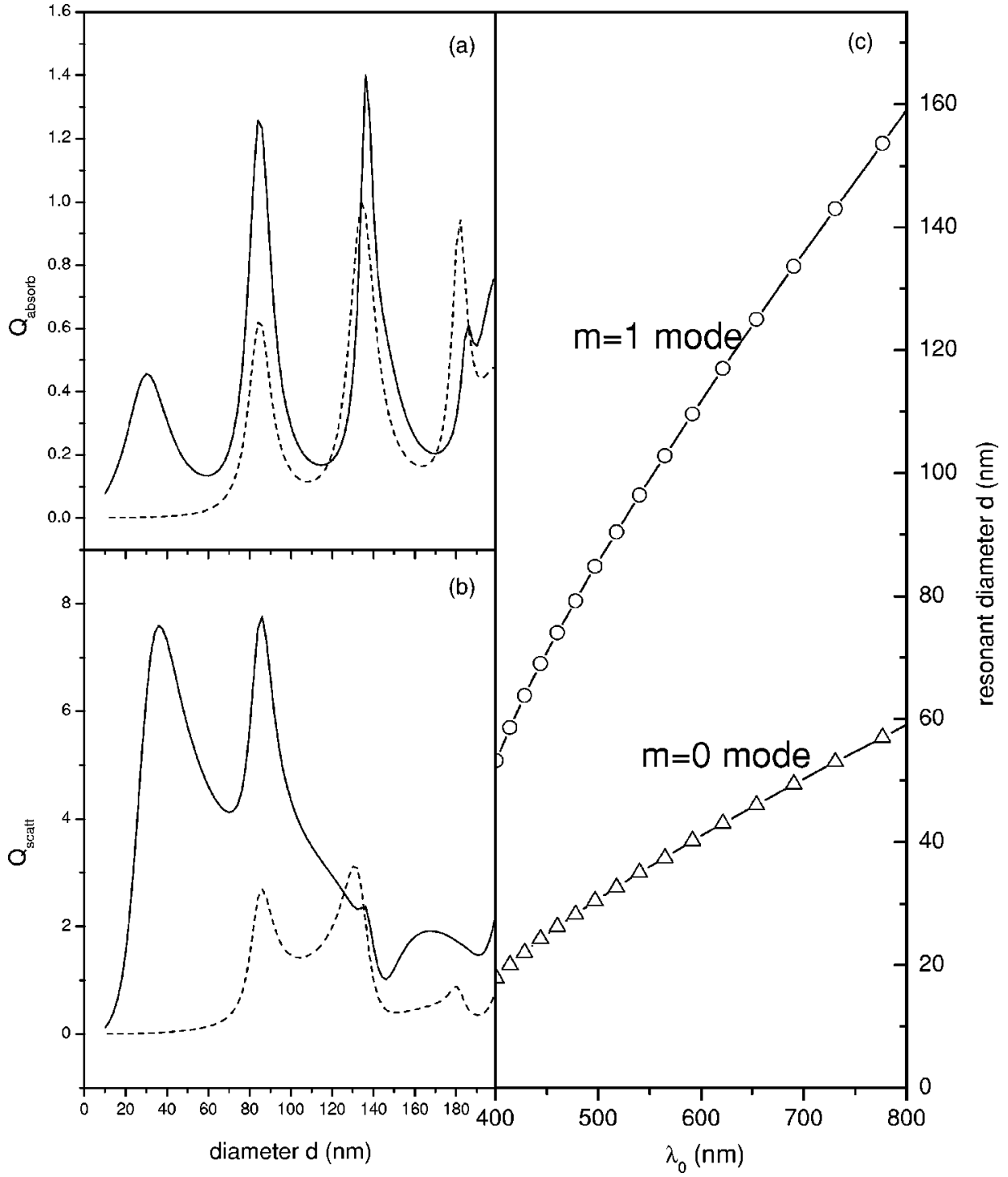

FIG. 3. (a) The absorption efficiency $Q_{a b s o r b}$ vs the diameter $d$ of Si nanowire. (b) The scattering efficiency $Q_{\text {scatt }}$ vs the diameter $d$ of Si nanowire. The incident light energy $h v=2.5 \mathrm{eV}$. The solid line is for the $S$ wave and the dashed line is for the $P$ wave. (c) The resonant diameter $d$ for $m=0$ and $m=1$ modes in the visible-light wavelength regime. light with energy $h v=2.5 \mathrm{eV}$ the first absorption peaks for both the TE and TM modes are around $d=100 \mathrm{~nm}$, so that the $m=0$ resonance mode at small diameter $d$ for Si nanowire is unique to its cylindrical structure of wire. ${ }^{8}$ The small value of diameter at resonance is essential for achieving high temperature by flashing of a photographic light.

\section{THE TEMPERATURE RISE OF SI NANOWIRES CAUSED BY VISIBLE-LIGHT ABSORPTION}

We now turn to consider the temperature increase induced by the absorption of incident light from the photographic flash. Following Ref. 2, the temperature rise $\Delta T$ of a Si nanowire can be calculated as

$$
\Delta T=\frac{I_{0} \sigma_{a b s o r b}}{\rho C_{p} V},
$$

where $I_{0}$ is the intensity of light, $\rho$ is the density of $\mathrm{Si}, C_{p}$ is the specific heat of $\mathrm{Si}$, and $V$ is the volume of the $\mathrm{Si}$ nanowire per unit length. For wavelength of light beyond $800 \mathrm{~nm}$, the absorption efficiency is rather small so that we can ne- glect the absorption of light in this regime. We assume that total light intensity $I_{0}=0.1 \mathrm{~J} / \mathrm{cm}^{2}$, and it is uniformly distributed between 400 and $800 \mathrm{~nm}$ (the energy is about $1.5-3.1 \mathrm{eV})$. We calculate the absorption cross section for incident light with fixed energy, and then integrate over the energy range $h v=1.5-3.1 \mathrm{eV}$ to obtain the averaged total absorption cross section and the corresponding temperature rise $\Delta T$ using Eq. (5). The result of temperature rise $\Delta T$ as a function of nanowire diameter $d$ is shown as the solid line in Fig. 4(a). We take the parameters $\rho=2.33 \mathrm{~g} / \mathrm{cm}^{3}, C_{p}$ $=0.713 \mathrm{~J} / \mathrm{gK}$. We note that the highest temperature rise $\Delta T$ is attained when the diameter of nanowire is around $d$ $=20 \mathrm{~nm} . \Delta T$ decreases rapidly when $d>20 \mathrm{~nm}$, and has a local minimum as $d \approx 40 \mathrm{~nm}$. This result agrees well with the experimental finding that the ignition of nanowire is easy for diameters less than $40 \mathrm{~nm}$. In Fig. 4(a), we also plot the calculated results for oblique incidence of light. The analytical formula needed for calculating the absorption efficiency $Q_{a b s o r b}$ in the oblique incident case can be found in Ref. 6, and will not be reproduced here. As the angle of oblique incidence increases, the temperature rise $\Delta T$ decreases and the peak structure at diameter $d=20 \mathrm{~nm}$ will be gradually 


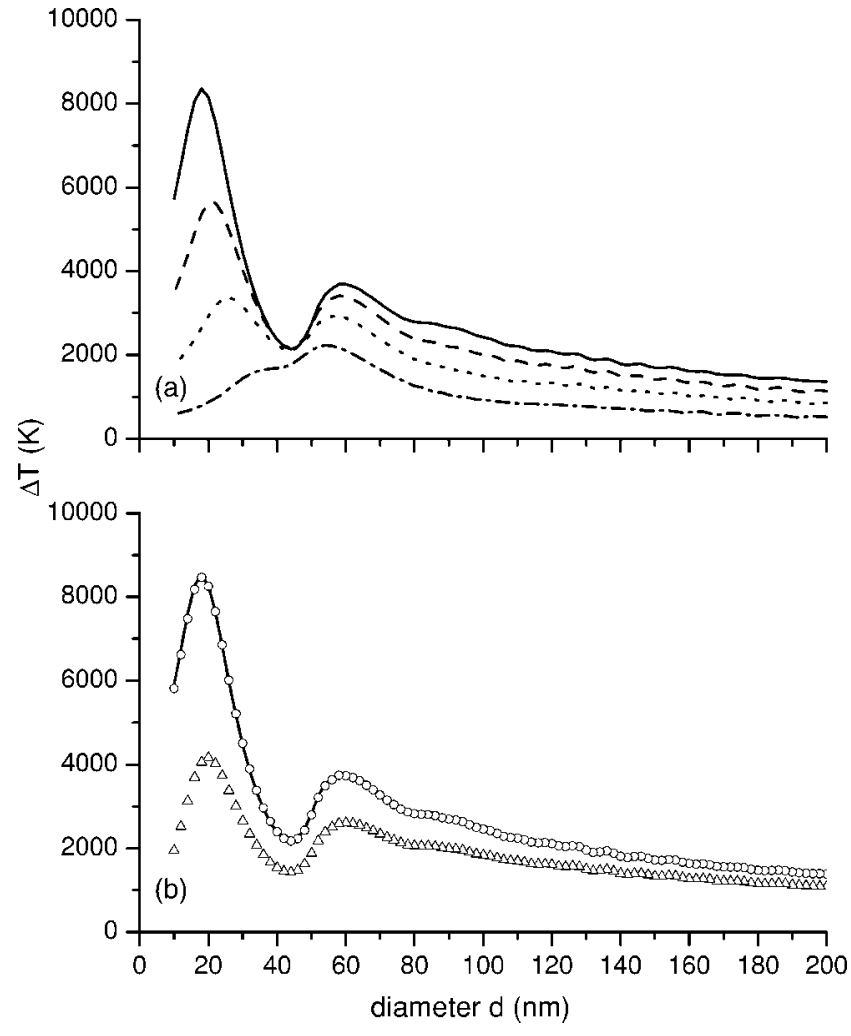

FIG. 4. (a) The temperature increase $\Delta T$ as a function of the diameter $d$ of $\mathrm{Si}$ nanowires. The solid line is the normal-incidence case $(\theta=0)$, and the dashed line $(\theta=\pi / 6)$, dotted line $(\theta=\pi / 4)$, and dash-dotted line $(\theta=\pi / 3)$ are oblique incidence cases. (b) The solid line assumes that the photoenergy absorbed from the flash is totally converted to heat. The open circles correspond to the results if the nanowires are coated with $2 \mathrm{~nm}$ of silica $(\epsilon=2.2)$. The triangles correspond to the result calculated by taking heat dissipation into consideration (see text).

smeared out. This is due to the fact that the coupling of light with the $m=0$ mode decreases when light is incident in a more oblique angle. The highest temperature rise can be achieved in the normal-incidence case.

Equation (5) assumes that all the energy absorbed is converted into the internal energy of the Si wires. During the optical annealing process, the $\mathrm{Si}$ wires absorb energy from the visible light, but at the same time some of the energy will be dissipated to the surrounding. We first consider the energy dissipation by convective heat transfer to the air. We will solve this time-dependent heat-transfer problem by the Crank-Nicolson method ${ }^{9}$ and show that even if the energy dissipation is considered, there is still enough energy to melt the Si wires by light. In our calculation we have also taken into account that the Si wires are coated with a silicon oxide shell (about 2-nm thick), and assume that the pulse duration of light flash is $1 \mathrm{msec}$. The physical parameters are taken as follows: For Si wire the thermal diffusivity and thermal conductivity are $\alpha_{1}=0.895 \mathrm{~cm}^{2} / \mathrm{sec}$ and $\kappa_{1}$ $=1.48 \mathrm{~W} / \mathrm{cm} \mathrm{K}$, respectively. For silicon oxide $\left(\mathrm{SiO}_{2}\right) \alpha_{2}$ $=0.848 \times 10^{-2} \mathrm{~cm}^{2} / \mathrm{sec}$ and $\kappa_{2}=1.38 \times 10^{-2} \mathrm{~W} / \mathrm{cm} \mathrm{K}$. The convective heat transfer between the silica surface and the air follows the Newton's law $-\left.\kappa_{2}[\partial T(r, t) / \partial r]\right|_{\text {surface }}=h\left(T-T_{a}\right)$.
Here the heat-transfer coefficient $h=1 \times 10^{-3} \mathrm{~W} / \mathrm{cm}^{2} \mathrm{~K}$, and $T_{a}$ is the temperature of air. The results are shown in Fig. 4(b). We note that when the Si wire is coated by 2-nm silica the energy absorption from the visible light is essentially the same as that without coating. When the energy dissipation is taken into account, the temperature rise $\Delta T$ for small diameter nanowire decreases considerably. Now the highest temperature is about $4000 \mathrm{~K}$ for the nanowire with diameter $d=20 \mathrm{~nm}$, but it is high enough to melt or ignite the silicon core.

Next we consider the energy dissipation of the Si nanowire by thermal radiation. It is well known for bulk materials that the total power of thermal radiation is proportional to the surface area and its temperature dependence will follow the blackbody radiation case. It is more subtle for nanomaterial. We note that the Si nanowires melt at temperatures below $1500{ }^{\circ} \mathrm{C}$. Before melting, we expect from the Wien's displacement law $\left(\lambda_{\max } T=2.898 \times 10^{-3} \mathrm{mK}\right)$ that the dominant part of thermal radiation energy comes from infrared wavelengths much longer than the diameter of nanowire. Hence the Rayleigh theory should be valid in this case. In $k_{0} a \ll 1$ (also $\epsilon \approx 1$ ) limit, the scattering coefficient is $D_{0}=-i(\pi / 4)\left(k_{0} a\right)^{2}(1-\epsilon)$, and the absorption cross section per unit length is $\sigma_{a b s o r b}=\pi a^{2} k_{0} \operatorname{Im}(\epsilon)$. Thus for sufficiently small diameters, the absorption ability of nanowire is proportional to the volume and also to $1 / \lambda_{0}$, which will have important consequences on thermal radiation proper-
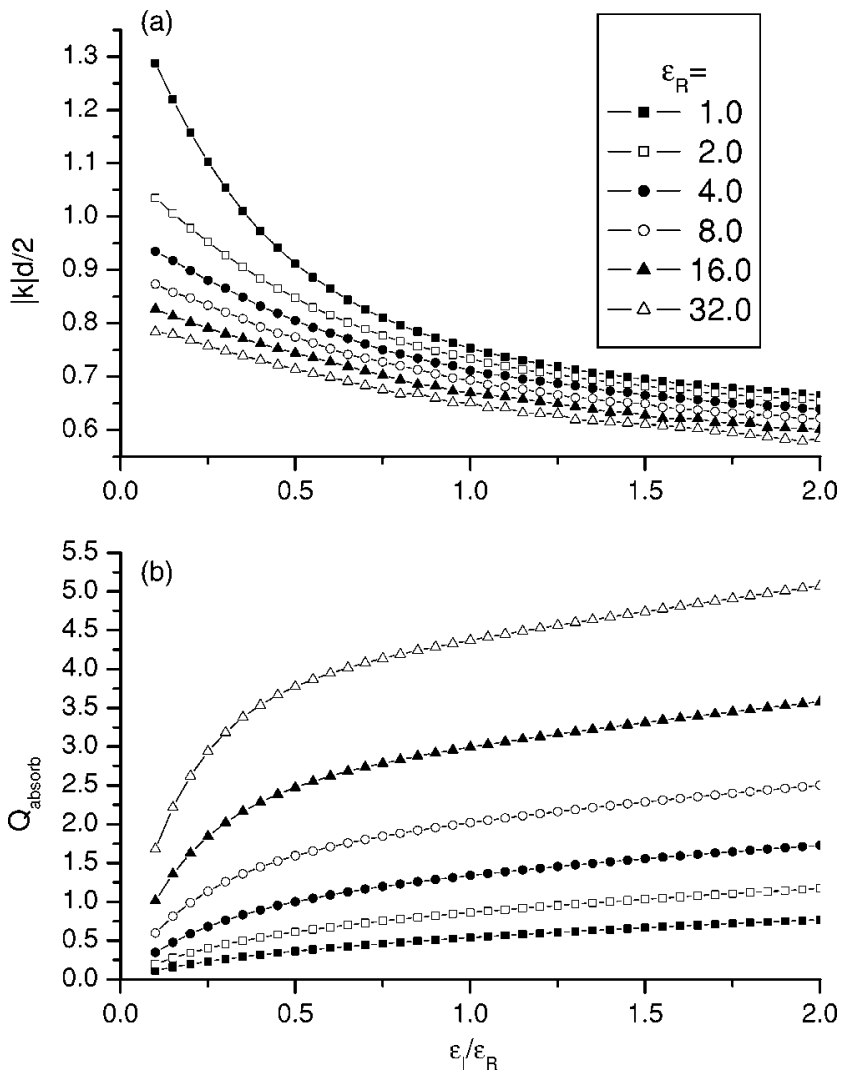

FIG. 5. (a) The diameter of nanowire with cylindrical geometry that achieves a maximum absorption in the $S$-wave mode with $m$ $=0$; where $k=k_{0} \sqrt{\epsilon}$ is the wave vector in the cylinder. (b) The corresponding absorption efficiency $Q_{a b s o r p}$ value at the peak position. 
ties, since according to the Kirchhoff's law for emission and absorption, we know that the emissivity of material is equal to its absorption efficiency. Thus for nanowire the total power of thermal radiation is given by the integral with frequency, $\quad P_{\text {radiation }}=\int_{0}^{\infty} \sigma_{\text {absorb }} n(\omega, T) d \omega$, where $n(\omega, T)$ $=\left(h \omega^{3} / 2 \pi^{3} c^{2}\right) 1 / \exp \left(\hbar \omega / k_{B} T\right)-1$ is the Planck function. We note here that $\sigma_{a b s o r b} / G=\pi / 2 k_{0} a \operatorname{Im}(\epsilon)$, where $G$ is the geometric cross section. Since both $k_{0} a$ and $\operatorname{Im}(\epsilon)$ are small at long wavelength for $\mathrm{Si}$ nanowires of $d=20 \mathrm{~nm}$, we can safely assume that the heat loss by radiation is very small. The nanowires are thus good absorbers of light at optical frequencies (note that at resonance $\sigma_{a b s o r b} / G>1$ ), but poor radiators of heat.

It may be interesting to go beyond our calculation for one specific material and see whether light can melt or ignite other nanowires. We shall consider the following problem: For a given permittivity $\epsilon=\epsilon_{R}+i \epsilon_{I}$ of nanowires of cylindrical geometry, what is the corresponding diameter of the cylinder which has a resonance in the $m=0$ mode of the $S$ wave? In Fig. 5(a) for several different values of the real part of permittivity $\epsilon_{R}$, the diameter of the cylinder at resonance is plotted as the function of the ratio $\epsilon_{I} / \epsilon_{R}$. The corresponding absorption efficiency $Q_{a b s o r b}$ at the resonance peak is plotted in Fig. 5(b). Figure 5 provides a "map" from which we can see when optical ignition or optical annealing will be applicable to nanowires.

\section{SUMMARY AND DISCUSSIONS}

In summary, we found that the apparently surprising optical ignition of $\mathrm{Si}$ nanowires can be explained entirely within the domain of classical Maxwell theory. One would normally expect that nanowires, because of their small diameter compared with visible wavelength, are far from a blackbody, and cannot absorb much light; hence the optical ignition is a surprise. But suppose that the nanowire is a blackbody. Then, the energy it absorbs per unit length is proportional to its radius $(r)$, while the number of atoms that shares the absorbed energy $\sim r^{2}$ per unit length. The temperature rise should then be $\sim 1 / r$. Small diameter nanowires should be heated up more than large ones if they are blackbodies. What we have shown here is that Si nanowires can have an absorption cross section that is even larger than the geometric cross section, due to a $m=0$ resonance that is unique to the cylindrical geometry of the nanowires. This explains the optical ignition phenomena. Since the model used is a simple one, based on a cylindrical coated nanowire in air, there might be other heat conduction channels so that the final temperature could be lower than what we predict in our model. In that case the absorption of light energy would have to be even more efficient than what we have calculated here in order to induce pyrolysis. Our work shows that the mechanism of resonant absorption constitutes at least one of the most important elements in nanowire light absorption and heating. The strong ability of nanowires to absorb light may have important applications in areas such as solar energy and smart ignition.

\section{ACKNOWLEDGMENTS}

We thank Professor N. Wang, Dr. L. Zhou, and Dr. X.H. $\mathrm{Hu}$ for useful discussions. This project is supported by Hong Kong RGC through Grant No. 600403.

\footnotetext{
*Corresponding author. Email address: phchan@ust.hk

${ }^{1}$ A. M. Morales and C. M. Lieber, Science 279, 208 (1998).

${ }^{2}$ N. Wang, B. D. Yao, Y. F. Chan, and X. Y. Zhang, Nano Lett. 3, 475 (2003).

${ }^{3}$ P. M. Ajayan, M. Terrones, A. de la Guardia, V. Huc, N. Grobert, B. Q. Wei, H. Lezec, G. Ramanath, and T. W. Ebbsen, Science 296, 705 (2002).

${ }^{4}$ B. Bockarth, J. K. Johson, D. S. Sholl, B. Howard, C. Matranga, W. Shi, and D. Sorescu, Science 297, 192 (2002).

${ }^{5}$ N. Braidy, G. A. Botton, and A. Adronov, Nano Lett. 2, 1277
}

(2002).

${ }^{6}$ C. F. Bohren and D. R. Huffman, Absorption and Scattering of Light by Small Particles (Wiley, New York, 1983).

${ }^{7}$ D. E. Aspnes and A. A. Studna, Phys. Rev. B 27, 985 (1983).

${ }^{8}$ U. Schröter and A. Dereux, Phys. Rev. B 64, 125420 (2001); D. Taverna, M. Kociak, V. Charbois, and L. Henrard, ibid. 66, 235419 (2002).

${ }^{9}$ M. N. Özisik, Boundary Value Problems of Heat Conduction (Dover, New York, 1989) 